\title{
TOOLS OFTHE TRADE
}

\section{Landscape surveys from the sky}

High-resolution and high-accuracy airborne topographic surveys are vital for observing and measuring changes in landscapes. Traditionally, however, surveying techniques were expensive and required specialist photogrammetry knowledge, limiting data collection and therefore understanding of landscape processes. Yet in the last decade, Earth science investigations have been revolutionised by the emergence of Structure-from-Motion photogrammetry (SfMP).

Similar in concept to a stereoscope, SfMP produces 3D scenes of the landscape. The technique uses automated software and feature matching to overlap multiple photos - typically taken from drones or planes with handheld or mounted digital cameras - to generate rapid digital elevation models and landscape orthophotos (geometrically corrected aerial photographs) with high precision and accuracy. SfMP solves scene geometry and interior (principal point and focal depth) and exterior (position and orientation) camera parameters, producing 3D models that can be georeferenced using points in the landscape or image positions.

SfMP-generated models, orthomosaics, and DEMs can be used to noninvasively reconstruct most landscapes at scales from sub-metre to hundreds of kilometres,

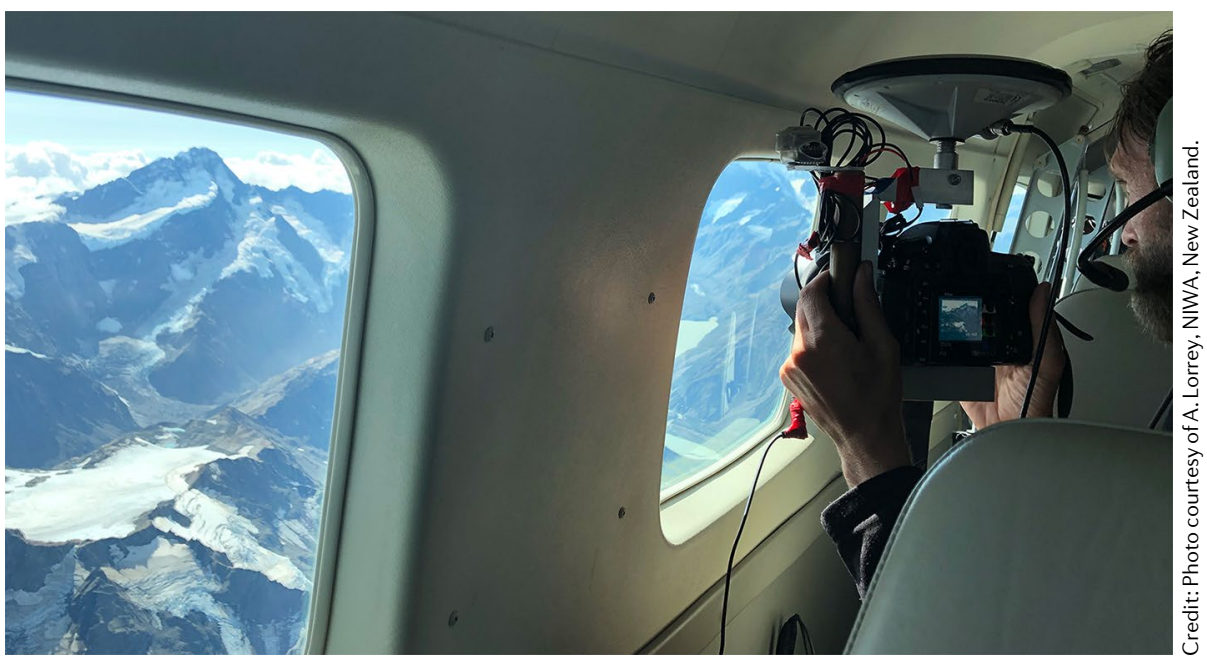

the resolution largely being controlled by camera pixels and the altitude from which the image is taken. Indeed, SfMP has been used for fine-scale measurements of coastal dune morphology to basin-scale documentation of landscapes and land use. Repeat surveys further enable changes to be quantitatively measured over various timescales, from near-instantaneous effects (such as hillslope erosion after severe rainfall) through to long-term changes (including glacier fluctuations associated with climate variability). In New Zealand, for example, annual
SfMP surveys have shown that glaciers have declined in area, length and volume since 2015. SfMP offers a powerful tool to understand landscape processes, but its use in geomorphological research requires robust surveying and adequate reporting to minimise uncertainties, reduce bias and fully realise its potential.

Lauren J. Vargo Antarctic Research Centre, Victoria University of Wellington, Wellington, New Zealand. e-mail: lauren.vargo@vuw.ac.nz The author declares no competing interests. 